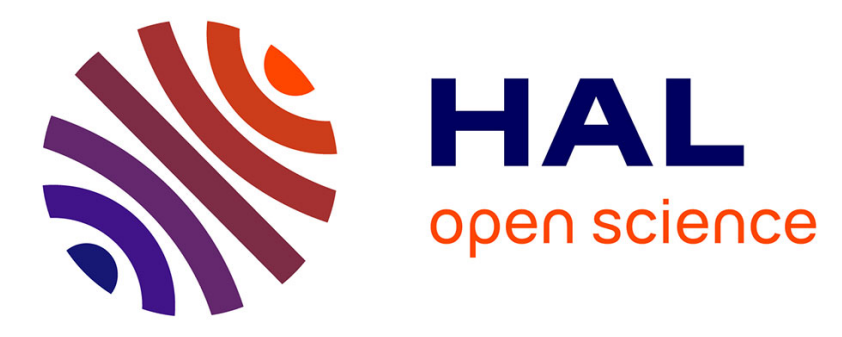

\title{
Theory of Constraints Thinking Processes on Operational Lean Programs Management Improvement: An Energy Producer Company Case
}

\author{
Margarida Gaspar, Luis Cristovão, Alexandra Tenera
}

\section{To cite this version:}

Margarida Gaspar, Luis Cristovão, Alexandra Tenera. Theory of Constraints Thinking Processes on Operational Lean Programs Management Improvement: An Energy Producer Company Case. 10th Doctoral Conference on Computing, Electrical and Industrial Systems (DoCEIS), May 2019, Costa de Caparica, Portugal. pp.125-142, 10.1007/978-3-030-17771-3_11 . hal-02295225

\section{HAL Id: hal-02295225}

\section{https://hal.inria.fr/hal-02295225}

Submitted on 24 Sep 2019

HAL is a multi-disciplinary open access archive for the deposit and dissemination of scientific research documents, whether they are published or not. The documents may come from teaching and research institutions in France or abroad, or from public or private research centers.
L'archive ouverte pluridisciplinaire HAL, est destinée au dépôt et à la diffusion de documents scientifiques de niveau recherche, publiés ou non, émanant des établissements d'enseignement et de recherche français ou étrangers, des laboratoires publics ou privés. 


\title{
Theory of Constraints Thinking Processes on Operational Lean Programs Management Improvement: An Energy Producer Company Case
}

\author{
Margarida Gaspar ${ }^{1}$, Luis Cristovão ${ }^{2}$ and Alexandra Tenera ${ }^{3}$ \\ ${ }^{1}$ Industrial Engineering and Management Student, Faculty of Sciences and Technology \\ (FCT), Universidade NOVA de Lisboa, Caparica, Portugal, ms.gaspar@campus.fct.unl.pt \\ ${ }^{2}$ ToC Expert, Goldratt consulting, cristovl@hotmail.com \\ ${ }^{3}$ UNIDEMI, Department of Industrial and Mechanical Engineering, Faculty of Sciences and \\ Technology (FCT), Universidade NOVA de Lisboa, Caparica, Portugal, abt@fct.unl.pt
}

\begin{abstract}
Several theories and associated models are arising in the field of systems' continuous improvement, focused on structured solutions to face the internal or external factors that affect them. Within these theories stands out Theory of Constraints (TOC), proposed to manage the most relevant constraints that exist within an organization. In this case study, the TOC Thinking Processes approach will be applied to the Management of Operational Lean Programs, on a pilot investigation at the organization were the Case Study took place. This application aims to analyze their Lean Program's Management in order to find the constraint that inhibits the system to reach its best level of performance, in order to support the development of robust improvement solutions that can solve the identified restrictions and sustain the proposed changes over time reaching a process of ongoing improvement.
\end{abstract}

Keywords: Theory of Constraints, Thinking Processes, Lean programs, continuous improvement.

\section{Introduction}

Nowadays, companies, whether on production or services fields, focus mostly on their levels of competitiveness on the global markets where they operate. So, they need to adopt strategies to guarantee their success amongst clients and stakeholders. The first step on choosing this strategy is to focus on the perception of the business processes flow [1]. On other hand, in order to step up the operational results, reduce costs and improve operations, it's usual to try implementing a culture of continuous improvement at the company as part of the business plan. One of these continuous improvement approaches is the Theory of Constraints that is considered by some as a management philosophy [2], [3] and by others as a methodology [4]-[6].

Theory of Constraints (TOC) was introduced to the scientific field on the 80's. Since then, it has been disseminated as a methodology or a philosophy to solve problems and 
improve systems' performance on many fields, by applying many structured TOC solutions. The less implemented solution of TOC is the Thinking Processes (TP), which can be verified by the published literature over the years. This solution was constructed to be applied mostly at complex systems, giving managers tools to think structurally about problems and how to solve them, providing sustainable solutions for organizations.

Thus, this research aims to give a contribution to the scientific field, providing a real Case Study developed at a top company, where the Thinking Processes tools were applied, showing their potential on an integrated application. This research effort intends to result on a valuable contribution to TOC-TP, providing increased results, since for the past 5 years, only few researches were published at the scientific level, as it will be exposed further. Notice also that on this Case Study, the application of TOCTP tools didn't follow its traditional approach, having been adapted to the company's reality, as it is stated further on chapter four. This adaptation proves that Thinking Processes tools can be orchestrated to answer any company's needs, providing adapted solutions with the same level of quality as any other continuous improvement approach. Also, considering all the revisited literature, this is one of the few Case Studies were all the tools were applied, showing the full potential of TOC-TP, not being limited to the application of one or two tools as a complement of a major analysis.

At an organizational level, the Case Study contributes to the company's Operational Lean Programs Management Improvement, introducing a new methodology, new improvement tools and a systematic approach to examine problems, turning this into a pilot investigation at the organization. What is unique about this investigation, regarding to the organization's reality, is the attempt to improve management practices inside a continuous improvement lean program itself. By doing so, it is possible to question why the management of a lean program is not as lean as it should be. As stated by Taveira [7] on a previous evaluation of the system, the company where the Case Study took place, saw lean as a mean to reduce the expenses and wasn't considering other major benefits on their daily routine and on its implementation and, nowadays, the problem seems to persist. With this research we aim to give the company a systematic reflection approach to analyze an organizational system and come up with robust solutions to improve it, applying all known Goldratt's TOC-TP tools, which rarely are put into practice as a whole.

Therefore, the main research question brought to discussion is: how a lean organization can benefit from TOC, and how it can provide reasonable and logic solutions for the effective and efficient improvement of the Operational Lean Programs Management? In order to answer the question, this paper is divided into five sections. The present section provides general insights about the Case Study, the subject's main highlights and the major research contributions, the following section explains the relationship of the paper to industrial and service systems, followed by a literature review on the TOC-TP topic on section number three. The fourth section details and explains how the Case was conducted and developed. The last chapter provides the discussion and conclusions about the obtained results, ending with some suggestions of further work on the field. 


\section{Subject Relationship to Industrial and Service Systems}

Planning is the core phase of a Plan-Do-Check-Act (PDCA) cycle, requiring a high level of effort. However, nowadays it is crucial to answer efficiently to the fast changes of global markets, and often organizations act before taking the suitable time to plan. By doing so, many companies fail on their plans and must rework their strategies, wasting resources and losing service quality. Thus, on industrial and service systems, when applying continuous improvement methodologies, such as Lean, Six Sigma or Theory of Constraints (TOC), it is fundamental to follow the inherent cycles of improvement, to assure the follow up of the planning as well as the fulfillment of the other phases of the cycles.

From the Theory of Constraints perspective, one of the most common applied tools among industrial and operations environments is the Drum-Buffer-Rope (DBR), which has been applied on many fields and types of industries. However, the same doesn't happen yet with the reflexive logical tool of TOC, the Thinking Processes, although it is recognized as the TOC tool with more improvement potential [8]. Its application to real industrial and service paradigms is not very wide and its potential as a continuous improvement methodology hasn't been fully explored.

The TOC Thinking Processes reflexive tools, when applied to an organization, imply systematic thinking following cause-effect logic and reflection mechanisms. By doing so, companies can track their organizational problems, think about solutions, construct future realities and come up with possible obstacles that they can discuss and solve before the implementation of the solution. Thus, the planning is all structured beforehand any operational action takes place, so organizations will be aware of which is the most effective plan they must stick to. This approach to planning will require a higher organizational interconnection of the players, to be supported on new levels of creative and intelligent business decision systems, to be able to produce less expensive, faster and valuable decisions for highly smart organizational decision making.

This Case Study tries to contribute to the expansion of Theory of Constraints Thinking Processes (TOC-TP) as a strong player on its methodological field, giving a full and updated analysis of how TOC-TP is applied nowadays and how it can be managed on a real and practical application on a service system. By applying TOC-TP to an energy producer company, that has many years of experience on lean programs, it is intended to show how an organization can benefit from TOC, and how it can provide reasonable and logic solutions for problems that tend to persist over the years, elevating its performance on lean applications.

\section{Theory of Constraints Thinking Processes (TOC -TP)}

\subsection{Theory of Constraints and Thinking Processes review}

In 1984, Goldratt published "The Goal" [9], a novel that introduces TOC to the management field, based on the story of Alex Rogo, his production plant and how he 
saves it from disaster with the help of his mentor Jonah. The basis of TOC goes by the premise that every system has at least one active constraint, which means, a systemic problem. On TOC way, constraints are seen as positive things, representing an opportunity to improve the given system. As the constraint sets the performance of the system, an improvement of it will improve the system's results.

In 1994, Goldratt also published "It's Not Luck", another novel which introduces the Thinking Processes and associated tools, trying to provide a systematic way to address the identification and resolution of problems concerning management policies [10]. According to Cox and Robinson [11], Goldratt proposed Thinking Processes as a way to apply the scientific method to the resolution of business problems, seeking to identify and analyze the inherent simplicity of a system through cause-effect logic. So, the use of the Thinking Processes tools should contribute to achieve system's improvements, to search for a graphical perception of its logical relations and to adopt a simple method to identify, analyze, understand and communicate problems, as well as develop logical solutions.

The identification of the constraint is very important, particularly when it is a nonphysical/intangible one because physical constraints are more tangible and much easier to identify and address. Also notice that many times just by changing relevant policies or performance measures, the existing main constraints can disappear, which results on exponential benefits towards the system's goal [12]. So, for this to happen, and to ensure a significant state of improvement on a system, we should answer three Goldratt's basic improvement questions, i.e. the TOC fundamental questions [13,14]: What to change? What to change to? How to change?, which later were expanded by Cox et al. [15] to the current five: Why to change? What to change? What to change to? How to change? How to measure and sustain the change? So, while answering these questions, TOC's continuous improvement actions should be developed according to the identified main constraint of the system [12], thought a prescribed process of continuous improvement (POOGI), consisting in Five Focusing Steps (5FS): 1) identify the system constraint, 2) decide how to exploit the constraint, 3) subordinate the rest of the system to the identified constraint, 4) elevate the constraint and 5) return to step one and don't allow inertia to become the constraint [13]. As the three basic improvement questions evolved, on the TOC's 5FS were added two pre-requisites processes to implement improvement actions, as it will be considered at the present research. So, according to Pass and Ronen [5], before the identification of the constraints we should also: 1) Stablish what is the organization's goal and 2) Define global performance metrics.

\subsection{Overview of Thinking Processes' (TP) recent literature}

The TOC Thinking Processes unlikely other TOC tools, as DBR, isn't so deeply developed in terms of practical and real situations. As referred at section one, to check the scientific current research level of the TOC-TP approach, a bibliography study was conducted, using research engines such as B-ON and Scopus. This research was developed considering the periods of time between 2008-2013 and 2014-2019 and defining a set of terms to conduct the investigation, using quotation marks to restrict joint terms such as "Thinking Processes". Also, to identify the scientific works directly 
related to the main subject (TOC) and the Goldratt term was also added to each research term. On Table 1, the results are identified on the research terms by "terms" and "Goldratt" search results. Also, in the scope of this search all sources and all types of information available were included, regarding to keywords and titles of conferences, papers, academic and scientific journals, reports and books.

Table 1. Theory of Constraints publications of the last seven and five years

\begin{tabular}{|c|c|c|c|c|c|}
\hline \multirow{3}{*}{$\begin{array}{l}\text { Research } \\
\text { Engine }\end{array}$} & \multirow{3}{*}{ Research Term } & \multicolumn{4}{|c|}{ Number of Publications } \\
\hline & & \multicolumn{2}{|c|}{$2008-2013$} & \multicolumn{2}{|c|}{ 2014-2019 } \\
\hline & & By keywords & By title & By keywords & By title \\
\hline \multirow{6}{*}{$\mathrm{B}-\mathrm{ON}$} & $\begin{array}{l}\text { Theory of Constraints/ } \\
* \text { Goldratt }\end{array}$ & $2735 / * 141$ & $160 / * 47$ & $2453 / * 47$ & $170 / * 72$ \\
\hline & $\begin{array}{l}\text { Theory of Constraints } \\
\text { Case Study } / * \text { Goldratt }\end{array}$ & $2 / * 0$ & $6 / * 4$ & $1 / * 0$ & $8 / * 2$ \\
\hline & $\begin{array}{l}\text { Theory of Constraints } \\
\text { Thinking Processes/ } \\
\text { *Goldratt }\end{array}$ & $1 / * 0$ & $2 / * 2$ & $0 / * 0$ & $0 / * 0$ \\
\hline & $\begin{array}{l}\text { Thinking Processes } \\
\text { Case Study/*Goldratt }\end{array}$ & $0 / * 0$ & $2 / * 1$ & $0 / * 0$ & $3 / * 0$ \\
\hline & $\begin{array}{l}\text { Thinking Processes/ } \\
*_{\text {*Goldratt }}\end{array}$ & $3 / * 0$ & $32 / * 3$ & $3 / * 0$ & $28 / * 1$ \\
\hline & $\begin{array}{l}\text { Thinking Processes } \\
\text { Tools/*Goldratt }\end{array}$ & $1 / * 0$ & $12 / * 3$ & $0 / * 0$ & $8 / * 1$ \\
\hline \multirow{6}{*}{ Scopus } & $\begin{array}{l}\text { Theory of Constraints/ } \\
\text { *Goldratt }\end{array}$ & $310 / * 205$ & $201 / * 86$ & $253 / * 184$ & $95 / * 74$ \\
\hline & $\begin{array}{l}\text { Theory of Constraints } \\
\text { Case Study/*Goldratt }\end{array}$ & $2 / * 2$ & $5 / * 5$ & $4 / * 3$ & $10 / * 8$ \\
\hline & $\begin{array}{l}\text { Theory of Constraints } \\
\text { Thinking Processes/ } \\
\text { *Goldratt }\end{array}$ & $17 / * 15$ & $5 / * 5$ & $10 / * 9$ & $4 / * 4$ \\
\hline & $\begin{array}{l}\text { Thinking Processes } \\
\text { Case Study/ *Goldratt }\end{array}$ & $2 / * 1$ & $3 / * 2$ & $4 / * 3$ & $3 / * 1$ \\
\hline & $\begin{array}{l}\text { Thinking Processes/ } \\
*_{\text {Goldratt }}\end{array}$ & $174 / * 18$ & $53 / * 10$ & $124 / * 14$ & $75 / * 10$ \\
\hline & $\begin{array}{l}\text { Thinking Processes } \\
\text { Tools/*Goldratt }\end{array}$ & $36 / * 11$ & $13 / * 7$ & $19 / * 3$ & $11 / * 3$ \\
\hline
\end{tabular}

Analyzing Table 1, regarding to B-ON search, it's possible to verify that, generically, Theory of Constraints has been more explored on recent years, unlikely Thinking Processes, which numbers decreased over the past few years. Regarding Case Studies, they have been more publication between 2014 and 2019 than between 2008-2013, but when adding the term "Goldratt" the number of available publications decreased to less than half on most cases. The same happens seems to happen for Thinking Processes Case Studies. Overall, although with few results were found, the research conducted by B-ON shows some consistency on the number of publications related to Theory of Constraints and Thinking Processes over the past 10 years, even though there's no results on some cases, according to the researched terms.

Regarding to the investigation conducted in the research engine Scopus, it is possible to find that numbers aren't much different from the scenario provided by B-ON, although Scopus provides a bigger amount of publications on every aspect (possibly 
because of research options provided by both engines). Generically, the number of publications also increased over the recent years as on the case of Case Studies publications. Although TOC publications numbers are still low, comparing to other continuous improvement methodologies, as Lean or Six Sigma. Concerning the identified Case Studies on Thinking Processes we could highlight the "Revolutionizing blood bank inventory management using the TOC thinking process: An Indian case study" (2017) by Lowalekar and Ravi [16], "The thinking process of the theory of constraints applied to public healthcare" (2019) by Bauer, Vargas, Sellitto, Souza and Vaccaro [17], "A process improvement approach based on the value stream mapping and the theory of constraints thinking process" (2014) by Librelato, Lacerda, Rodrigues, Veit [18].

Although Scopus provides a higher number of publications on most cases and having in consideration the cited publications, the reduced research on TOC-TP remains, providing the need to increase research and applications on this subject and on TOC area in general.

\subsection{Overview of Thinking Processes tools}

Goldratt developed a set of Thinking Processes tools to put TOC-TP on practice. The following tools were developed in a format of logic trees and a cloud: Current Reality Tree (CRT), Evaporating Cloud (EC), Future Reality Tree (FRT), Negative Branch Reservation (NBR), Prerequisite Tree (PRT) and Transition Tree (TT). Later, Dettmer [19] also added the Goal Tree (GT) and Goldratt as well added, the Strategic and Tactic Tree (S\&T), which is today the last contribute for the TP tools and it is also considered the last tool of the sequence that should be implemented. Figure 1 represents the existent connection between the five questions, the inherent application of the five steps and the TP tools and how they all work together to establish a full practical investigation on the Theory of Constrains Thinking Processes.

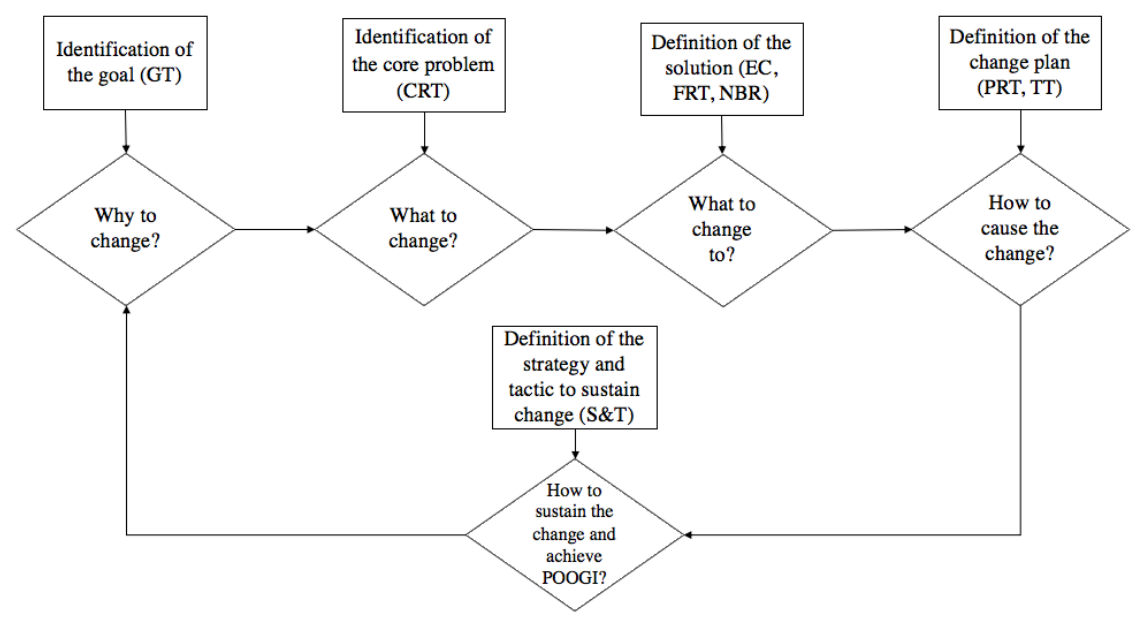

Fig. 1. Framework of the TOC-TP tools application 
Concerning to the roles of TP tools, the Goal Tree answers the first question "Why to change?", identifying the organizational model proposed to achieve the goal. Before a company can focus, it is necessary to have in mind what is the goal. Once the answer gets clear, the Necessary Conditions (NC) and Critical Success Factors (CSF) to achieve the goal are defined to identify which actions will be used to manage the progress. For the second question, CRT answers the question "What to change?", which allows to diagnose what, in the system, needs to be changed [4], which is a logical structure designed to illustrate the actual state of the system, isolating what needs to change, identifying the major problems (the undesirable effects, UDE) and draw the path to the root causes and the core problem.

Once the CRT is fully designed, the constraint emerges and the best way to manage it is through commitment. So, in order to solve the core problem, TOC defines that behind every problem there is a conflict that blocks any robust solution. In order to evaporate this conflict and get plausible solutions (the injections) Goldratt suggests the $\mathrm{EC}$ as the perfect TP tool to serve this purpose, answering the question "What to change to?" [4].

The fourth tool of the TP set is the Future Reality Tree (FRT) which allows to construct a solution that, when implemented, replaces the Undesirable Effects (UDE) that are the basis for the CRT, by Desirable Effect (DE) in the FRT. The FRT is designed to ensure that all UDE will be eliminated by the solutions founded at the Evaporating Cloud (EC), providing a valid alternative for the future. However, when trying to get buy-in of the system's owners it's crucial to make sure that there is no potential resistance related with the proposed solutions. This type of thought is considered when referring to the Negative Brunch Resolution (NBR), which determines if there are negative branches on the new solutions. By trimming these branches with new injections, should be possible to come out with a more robust solution for the system we are trying to improve.

The next TP tool is the Prerequisites Tree (PRT) that aims to answer the question "How to cause the change?" by identifying the obstacles that prevent the chosen injection(s) from being implemented [4]. The transition Tree (TT) is also used to give specific actions to the injections implementations i.e. combining each provided effect successively with the subsequent specific actions to produce new improved effects, contributing to answer the same question as a PRT. According to Dettmer [19, 20], the PRT can be combined with the TT. Together they should be capable to provide a more powerful and robust tool to structure the injections that are going to be implemented on the future reality of the system, having into account the possible obstacles on the way and eliminating them.

The Strategic and Tactic tree (S\&T) is used to identify and communicate the identified strategies and tactics to ensure Management's attention at all levels to be synchronized and focused on the highest priority changes. This last tool has been specially designed to help Top Management prevent common mistakes that result in failures of change initiatives within organizations. It is possible to verify that for each strategy (S) there must be a tactic (T), so an S\&T tree consists of a number of pairs $S$ and $\mathrm{T}$, presented at various levels, each level represents one layer of responsibility within the organization [14]. 


\section{Applying TOC-TP to Operational Lean Programs Management}

\subsection{Background to the Case Study}

According to Saunders [21], the strategy to develop this investigation was defined as a Case Study, suiting the definition given by Robson [22]. This author defines a Case Study as a strategy for doing research which involves an empirical investigation of a particular contemporary phenomenon within its real-life context using multiple sources of evidence.

Thus, the company where the Case Study took place is part of a Group which the main business is the production, distribution and commercialization of energy. This company in specific is responsible to manage the production of thermic and hydric energy, on Production Centers across Portugal. Being decentralized, with more than 40 production points, it is difficult to manage all the operations. To avoid resources' waste (money, time, quality) and to be as more efficient as possible, in 2006, the company launched the Lean Program. First, this Program was a pilot on a chosen Center and then it was replicated to many others. Nowadays the Local Programs are at the sustention phase, being often monitored and evaluated, answering to the KPI that are suggested by the managers.

To evaluate the system "as-is" was necessary to interview the company staff based on a design thinking approach and on brainstorming. This evaluation, which details are referred at Table 2, was carried out by the company on a lean event. The evaluation was based on the steps of a lean initiative life cycle to understand both positive and negative opinions of the lean practitioners at each stage, also considering all the geographies where exists a Lean Program. Based on this empirical data, the first analysis to the system was made, based on the given negative feedback in order to analyze, at a first stage, the main problems of the system.

The data collected comes from various sources, which were combined to analyze the system, in order to get a fully understanding of it. They may include, for example, interviews, observation, documentary analysis and questionnaires [21].

Using tools such as Pareto Diagram and Ishikawa Diagram, it was possible to scrutinize a large amount of data. These tools allowed to group the main issues identified by the staff, in categories such as, Difficulty and Resistance to apply Lean, Unavailability of staff, Communication problems, which are the main concerns of the company. Later, these topics where addressed on the application of TOC-TP.

Table 2. Details of the Lean event

\begin{tabular}{|r|l|}
\hline \multicolumn{2}{|c|}{ Lean Day } \\
\hline Date & November 2017 \\
\hline Duration & One day \\
\hline People & Approx. 10 teams of 10 company employees (different roles) \\
\hline Roles & $\begin{array}{l}\text { Multiskilled employees (managers, technicians, engineers, } \\
\text { operational collaborators) }\end{array}$ \\
\hline Purpose & $\begin{array}{l}\text { Evaluate the status of Operational Lean Programs, based on staff } \\
\text { opinions }\end{array}$ \\
\hline
\end{tabular}




\begin{tabular}{|r|l|}
\hline Goal & $\begin{array}{l}\text { Improve the management of Operational Lean Programs and the } \\
\text { satisfaction of the staff with the Lean Program }\end{array}$ \\
\hline $\begin{array}{r}\text { Main } \\
\text { Approach }\end{array}$ & Design Thinking and Brainstorming \\
\hline $\begin{array}{r}\text { Type of data } \\
\text { collected }\end{array}$ & Empirical, collected by semi structured interviews \\
\hline
\end{tabular}

The following subsections show some diagrams that represent the trees and clouds developed for his problem as well as its explanation.

\subsection{Why to Change? The Application of the GT}

After the empirical analysis done to the data provided by the company, the main goal of the investigation was set to start the development of the TOC-TP logical tools. This is also considered the first step to develop the first Thinking Processes tool, the Goal Tree. Thus, with the agreement of the company, the main goal of the Case Study was defined as to identify the main actions to follow "to improve the effectiveness and efficiency of the Operational Lean Programs Management (OLPM)". In fact, the Goal Tree shows the main actions to have into account when trying to reach the proposed goal. The many inputs given by the company were crucial to identify which were the Critical Success Factors (CSF) and the Necessary Conditions (NC) that needed to exist to achieve the Goal. These factors and necessary conditions are defined to represent how the company must perform if the goal was achieved (although it hadn't been, due to the undesirable effects that create the gap between the reality and the desired state). The CFS are the fundamental aspects that are considered crucial to achieve the proposed Goal and the NC are the conditions that must exist to enable the Critical Success Factors. The organization's staff had an active role helping to construct the Goal Tree, as their opinions, feelings and inputs were collected from a brainstorming session. By actively construct the Goal Tree with the company, this tree can provide an accurate answer to the question to "Why to change?", giving the perception that the problem really exists and there's a need and a reason to create a positive change on the system. As the desired reality was constructed, the same session was used to inquire the participants about what is preventing the organization from achieving the Goal, constructing a list of 18 UDE. This list confirmed the main issues revealed on the first analysis to the system and those inputs were the basis of the following step: What to Change? That is explained in detail on the following section.

\subsection{What to Change? The Application of the CRT}

The reverse line of thought was taken into account to think about the undesirable effects (UDE) that prevent the system from achieving the desired state. Between the eighteen UDE identified on the system, the CRT (Figure 2) shows the situation as perceived at the time, answering to the question "what to change?". This diagram was constructed, from top-down and its read bottom-up, linking all the intermediate effects to the UDE, using inputs like the data analysis and team brainstorming, to develop logical cause- 
effect connections. At CRT, these cause-effect connections use "IF...THEN" statements, for example IF "Lean Programs aren't included in the QMS" OR "Improvement is seen as a time consumer" THEN "Improvement isn't considered as a management system".

Analyzing the CRT is possible to state that the intermediate effects that connect UDE are numbered above the boxes and UDE are identified by their numbers (e.g. UDE 1.2). Therefore, the entities " $A$ ", " $B$ ", " $C$ " and "L" represent long connections between entities. For example, entity "A" means that UDE 5.2 is connected to the UDE 1.1 by the ellipse (which represent an "AND"). However, "L" means that there is a negative reinforcing loop at the tree, meaning that when connecting those effects, repeated negative consequences occur at a faster pace.

From Figure 2 it is also possible to see that the main problems on the system are related to "Training", "Means to report lean initiatives" and "Alignment of metrics" factors, which are the identified Critical Root Causes (CRC). These CRC can by identified by spotting the UDE that have no entry connections (UDE 4.1) and by counting which UDE connects with most of other entities at the tree, for example, UDE 5.2 and UDE 5.3. These CRC are the problems that mainly contribute to the existence of core problem identified at the CRT. Therefore, these CRC must be scrutinized to be evaporated by EC. Not surprisingly, the major problem (core problem) that worried all participants including managers was, still, the "Lack of Commitment with Lean and Lean's culture", which is consistent with the problem found by Taveira [7]. This entity is represented at the bottom box of the CRT (box 55), has no entry connections and leads to most of the UDE (directly or indirectly) from bottom to top until reaching the Goal (the upper entity).

By developing the CRT, it is possible to conclude that a major and transversal problem has been affecting the company and the management of Lean Programs over the years and there wasn't no solution at that time. How can an organization be Lean and have a healthy Lean Program if it isn't felt on their culture and on a daily basis? How can a long term Lean Program have such structural problems as training, reporting and metrics? These paradigms were taken into consideration from the application of the CRT until the end of the research to suggest ways to solve it. The identified core problem becomes the focus of the cloud EC, revealing the dilemmas. 


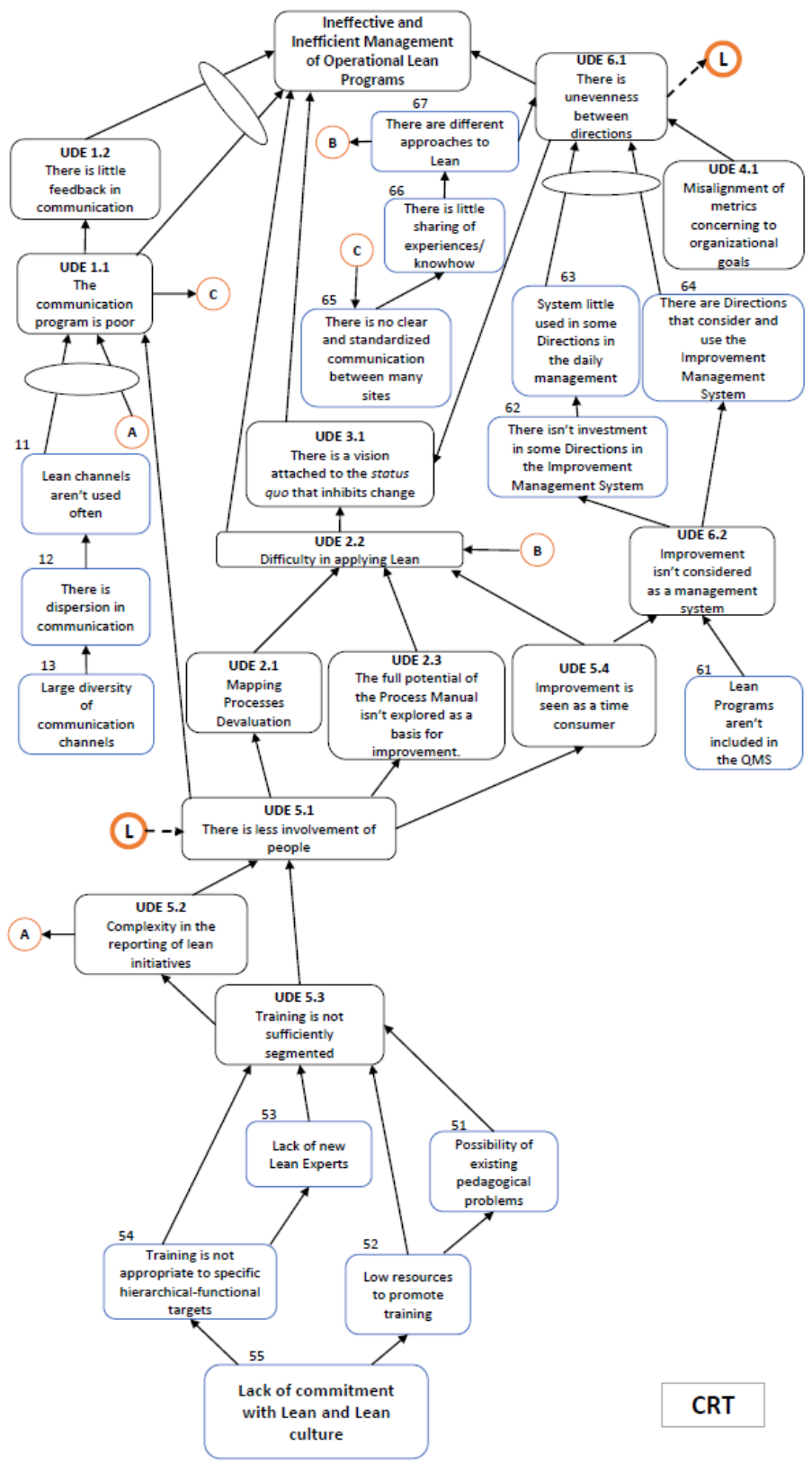

Fig. 2. Current Reality Tree 


\subsection{What to Change to? The Application of EC, NBR and FRT}

After the analysis of the CRT we are now able to study more deeply each of the main problems identified on the system applying the EC, NBR and FRT. The combination of these tools answers the third question "What to Change to?", giving a possible reality of the system after the change. Also, on this phase it is possible to come up with suitable new injections to mitigate the systemic problems.

To apply the Evaporating Cloud and to clearly point the dilemmas or conflicts regarding to the TOC-TP language, four clouds were developed, one of them to the core problem and the others to each of the identified CRC. Figure 3 represents the relationship between all the developed clouds, which focus is identical and represents the proposed Goal exposed at the first diagram. So, it is possible to state that entity A is the same for every cloud, representing the "Effective and efficient management of Operational Lean Programs".

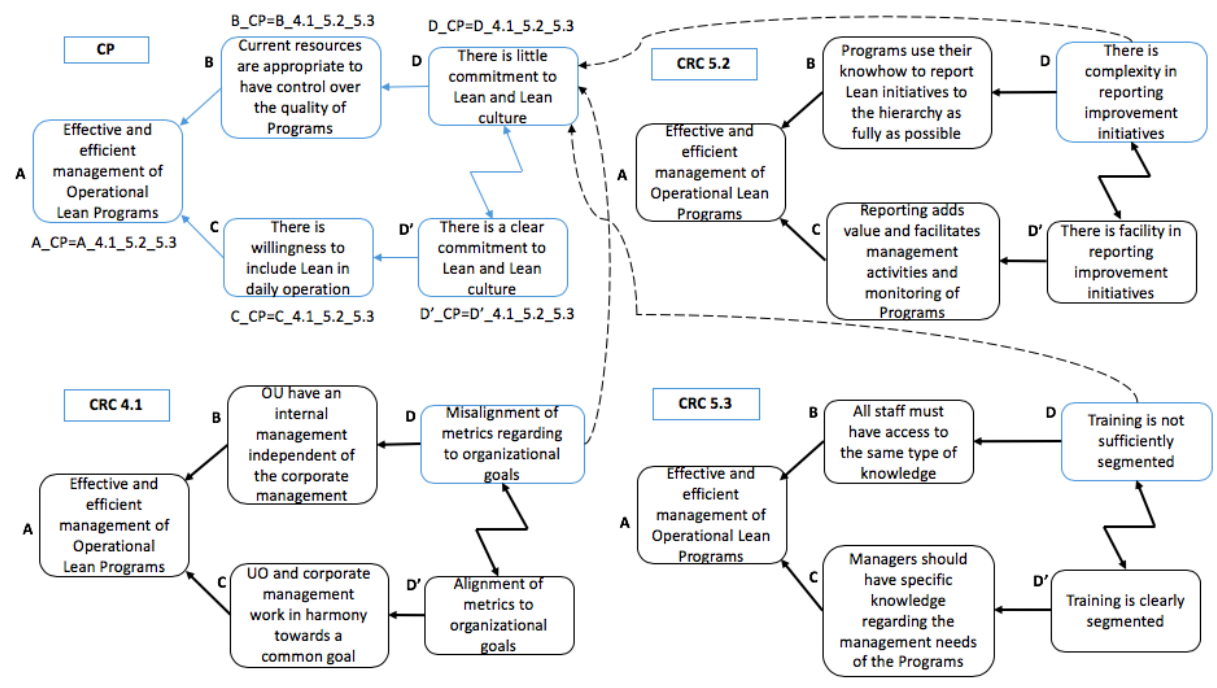

Fig. 3. Evaporating Clouds (EC)

As constructing each cloud, we can identify the weakest necessity cause linkage in the EC by creating assumptions that would be able to break it. These assumptions are created by necessity logical relations as "In order to have A, we need B". When having some assumptions for a given link it is necessary to find one statement weak enough to be broken by an injection that will dissolute the conflict. So, after the selection of the link to break, possible injections are created to evaporate the conflict and the cloud.

The "CP" cloud at upper left corner of Figure 3 represents the EC for the core problem. Its dilemma can be interpreted as: to have an "effective and efficient management of Operational Lean Programs" we need "willingness to include Lean in daily operation".

In order to have a "willingness to include Lean in daily operation" we need to obtain "a clear commitment with Lean and Lean Culture". On the other hand, for an "effective 
and efficient management of Operational Lean Programs" we need "the current resources to be appropriate to have control over the quality of Programs". And for this should be "little commitment to Lean and Lean culture" (since the current resources aren't considered enough). This is in direct conflict with the lower branch, which is represented by an elbow double arrow. Hence, we can't have much and low commitment with Lean at the same time.

At this Case Study, a total of 14 injections were brought to discussion with the representative organization manager to validate the findings. This process was repeated for every cloud and all the injections were analyzed with the support of an Efficiency/Difficulty matrix elaborated for the purpose, which was crucial to study the impact of each injection on the system. By this analysis, two injections were declined, four injections were approved by the organization to mitigate de training problem, four injections for the metrics issue and tree injections were proposed for the reporting. Although these injections will have an indirect effect on the core problem, contributing to its mitigation, one more injection was approved to directly mitigate the core problem.

Even though we are analyzing the EC, by chance, this application converged to what is called the Three Clouds Method, that is an alternative approach to the conventional application of the CRT [23]. At this investigation we chose to apply the conventional method of the CRT as we consider that is the most robust solution for the purpose. The advantage of having the architecture of the three-cloud method applied to the EC, as is represented at Figure 3, is that we can study the system as a whole by linking the entities on the clouds to each other's by their common points, as is shown by the arrows that converge from entity " $\mathrm{D}$ " to the entity " $\mathrm{D}$ " at the "CP" cloud.

The analysis of the EC concludes, as expected from the previous feedback, that the system needs a relevant improvement on what concerns to Lean commitment and cultural change. This change should be wider and deeper than just to mitigate the identified problems to align visions, practices, and to work in harmoniously on a Lean organization. However, we expect the proposed injections to help substantially on this matter.

After the final selection of the eligible injections to be implemented, the organization's manager selected the more volatile injection being "Linking programs and goals that translate into financial rewards/other benefits". This injection was scrutinized using the NBR as this tool that can induce negative side effects to the system. Next, NBR was trimmed by the division of the volatile injection into two "Use only non-financial and career progression benefits" and "Use other company benefits", that placed strategically at the NBR, could invalidate the negative effects, preventing the system from new UDE and the change, possibly, from failing.

The last step to finish answering the basic question "What to change to?" is the application of the Future Reality Tree. This tree comes up through the positive effects generated on consequence of the injections proposed at the EC, showing what would change if the system improved its effectiveness and efficiency on the management of Operational Lean Programs. Usually, these effects are the opposite of the negative effects identified at the CRT. So, the FRT shows, by drawing the logical paths, how the injections implemented on the system can create positive effects that will converge on the goal's achievement. 


\subsection{How to Change? The Application of the PRT+TT}

After the definition of the future reality it is necessary to induce the change by the application of the PRT and the TT, answering the question "How to change?". These two trees will be applied as a single tool, as justified by Dettmer [19], since the extension and complexity of the Case Study doesn't justify its independent utilization. Ideally, this tree would be constructed for each injection created previously, to analyze the details of its implementation on the system. However, on this paper we give only one example to illustrate how an injection can be explored by TOC-TP. Later, it is expected the company to construct the rest of the PRT+TT diagrams, to plan the implementation of the improvement actions.

Thus, a list was created to specify the intermediate objectives (IO) on the implementation of the injection. These intermediate objectives are the steps to follow to achieve the execution of the injection. Reading the diagram bottom-up, the IO are represented by boxes with curved edges, on a necessary condition thinking: "In order to have A we need B". For example, on Figure 4, "In order to analyze comparatively Lean tools with EDP Lean tools we need to Detail Lean tools according to using criteria". Then, another list was created to identify the obstacles regarding to the implementation of the chosen injection. These obstacles are assigned to the links that may have associated negative effects and can be interpreted as obstacles that prevent the system from the correct execution of the action. After, the PRT was created by linking the obstacles with the corresponding IO, as shown at Figure 4.

But, as stated before, this isn't the final stage of the tree, the next step is to trim those obstacles with specific actions (SA) to finish the implementation plan. These actions are necessary in order to prevent the improvement to fail and to avoid inertia. For example, to prevent the Obstacle 1 (O1) "Unawareness of the detail of the performance of functions" from happening the Specific Action 1 (SA1) "Contact the company and clarify any doubts that may exist" must be executed. Regarding the structure of the PRT+TT (see Figure 4), a plan of implementation for this specific injection was defined for the organization to follow. It is expected the organization to implement the rest of the injections taking into account the example given on the research and in order to promote a sustainable change, closing the first cycle of change by TOC-TP.

\subsection{How to Measure and Sustain the Change?}

To answer this question, at first, we would have to build a S\&T tree. But, considering the lack of enough knowledge by the research team about companies grounding on this subject to develop this tree, the research team decided not to pursuit with its construction. The S\&T tree was idealized to be implemented by managers, on project management subjects, as a tool to help them on the definition of the organizational strategy, which action is outside of the main scope of the present study. Nevertheless, S\&T should be implemented on projects and should be orchestrated by top management as a macro tool to manage the main lines of a bigger strategy. 


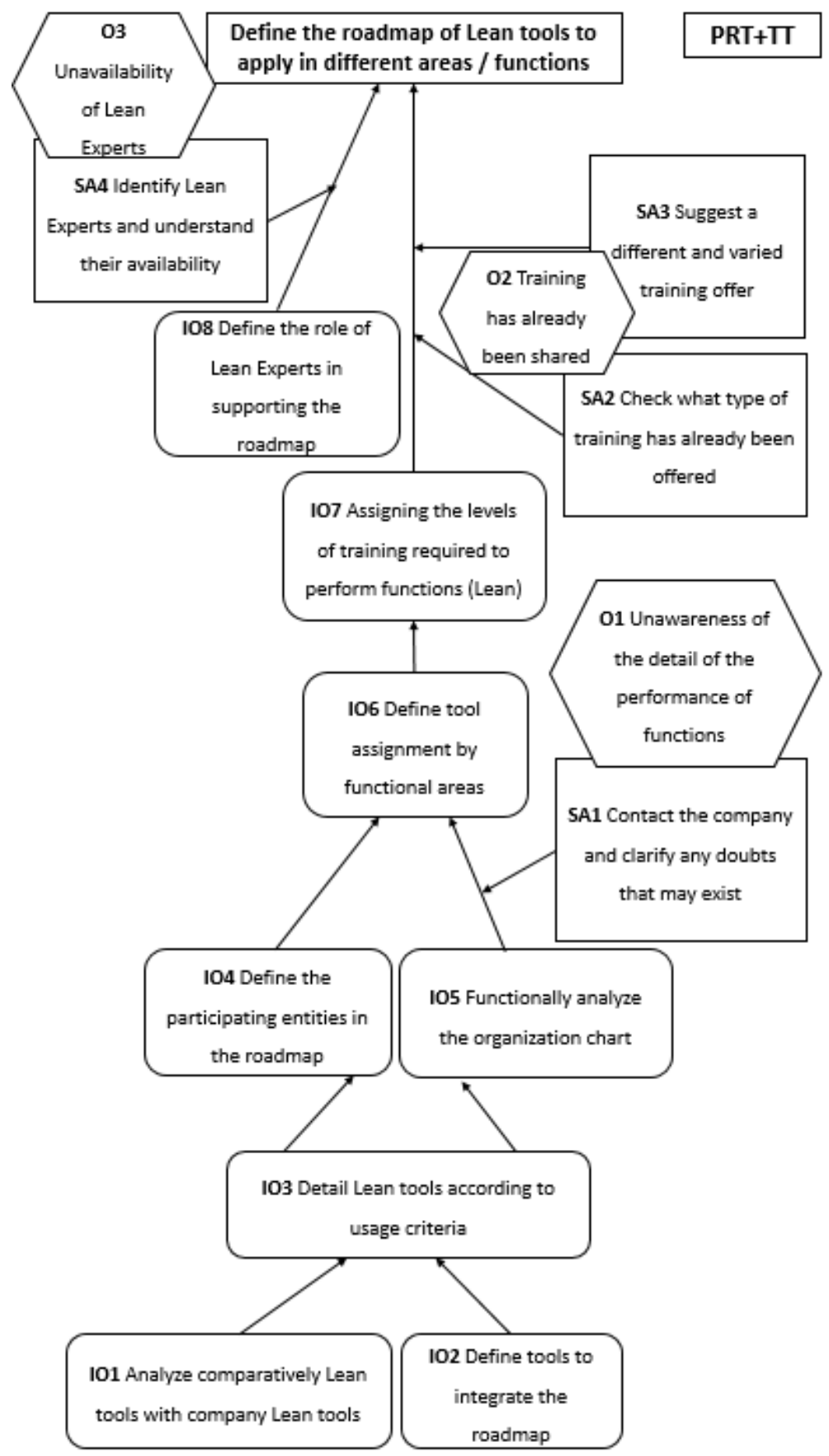

Fig. 4. $\mathrm{PRT}+\mathrm{TT}$ 


\section{Main Conclusions and Further Work}

This research allowed to consider solutions for an organizational problem that persists over the past years on a lean based company. The problem, lack of enough commitment with Lean and Lean's culture, is a long-term issue at the organization that is felt at the modus operandi of staff and at the organizational culture. In order to solve this problem, twelve injections were approved by the organization to put in practice. As an example, one of them was structured and prepared for the organization to implement.

The research allowed to implement GT to find the goal, the CRT to find the core problem, the EC to come up with injections, the NBR plus the FRT to structure and prepare the future reality, and the PRT+TT to give a figure of what one of the implementations could be. Given the full implementation of TOC-TP, the organization has now a systematic ground to promote the resolution of major problem that affects the Management of their Lean Programs. However, this Case Study doesn't validate the Theory and its suppositions, it just represents a contribution to the empirical application. Although the systematic method to apply Thinking Processes is concrete and has strict rules, its application is empirical and mostly qualitative, which produces qualitative results, that can have many interpretations. The lack of investigation on the field, doesn't provide yet a solid ground of exploration, giving few examples of application to better analyze systemic problems.

It is considered that the research question was mainly answered on its purpose as the research allowed to put into practice a full framework of basic TOC-TP tools, giving a sense of full accomplishment of the process of ongoing improvement, by using causeeffect logic and reflecting about what could be improved on a given system. However, the present investigation stills lacks on giving proof and analysis of the implementation of the injections on the company, measuring its results and, perhaps, starting another cycle of improvement to find if the problem sustains. Another limitation of this research approach is that the case study results can't be extrapolated to other realities since every company has a different reality. Due to the type of data collected and to the exposed problem on the Case Study, tools such as S\&T tree, TOC's Throughput Accounting and the verification of KPI weren't considered on this case study report, which could be developed in order to assure that, company could continually monitor the change and the solutions implementation impacts. Also, it is recommended to continually measure the satisfaction indexes of the staff regarding to lean, especially after the implementation of the improvement actions.

On this Case, TOC was used to provide a framework for the change needed on the system, regarding to the identified core problem and to provide a clear logic-based argument for the changes proposed, which are essential to ensure the sustainability of the Lean Programs. Also, this Case Study contributed with an innovative problemsolving method for the company and to increase TOC-TP exploration on the scientific field, contributing to encourage pairs to further explorations of TOC-TP. This research also adds to verify the potential and robustness of this method on smart organizational improvement, by giving unique and maybe out of the box solutions to organizations which require new levels of creative and intelligent business decision tools in order to be able to produce less expensive, faster and valuable decision i.e. highly smart organizational decision making, 


\section{Acknowledgements}

This work has been partially supported by UNIDEMI - Research and Development Unit for Mechanical and Industrial Engineering by the Portuguese FCT-PEST program UID/EMS/00667/2019.The authors are grateful to the supporter research company's collaborators which the researcher had the pleasure to work during the development of this research work.

\section{References}

[1] Z. T. Şimşit, N. S. Günay, e Ö. Vayvay, «Theory of Constraints: A Literature Review», Procedia-Social Behav. Sci., vol. 150, pp. 930-936, 2014.

[2] M. Gupta e L. Boyd, «Theory of constraints: A theory for operations management», Int. J. Oper. Prod. Manag., vol. 28, n. 10, pp. 991-1012, 2008.

[3] V. Sukalová e P. Ceniga, «Application of the Theory of Constraints Instrument in the Enterprise Distribution System», Procedia Econ. Financ., vol. 23, pp. 134-139, 2015.

[4] V. Mabin, "Goldratt's "Theory of Constraints" Thinking Processes: A Systems Methodology linking Soft with Hard», History, pp. 1-12, 1999.

[5] S. Pass e B. Ronen, «Management by the Market Constraint», Int. J. Prod. Res., vol. 41, n. 4, pp. 713-724, 2003.

[6] L. Taylor III e A. Rekha, «Applying Theory of Constraints principles and Goldratt's Thinking Process to the problems associated with inventory control», Franklin Bus. Law $J$. , vol. 2016, n. 4, pp. 83-104, 2016.

[7] A. Taveira, "Avaliação da Sustentação da Metodologia Lean numa Organização: Caso de Estudo na EDP Produção», Universidade Nova de Lisboa, 2015.

[8] E. M. Goldratt e J. Cox, The Goal: A process of ongoing improvement, 1st ed. North River Press, 1984.

[9] C. Chaudhari e S. Mukhopadhyay, «Application of Theory of Constraints in an integrated poultry industry», Int. J. Prod. Res., vol. 41, n. 4, pp. 799-817, 2003.

[10] J. F. Cox III e J. G. Schleier Jr, Eds., Theory of constraints Handbook. New York: McGraw-Hill, 2010.

[11] J. Cox III, L. Boyd, T. Sullivan, R. Reid, e B. Cartier, «TOCICO Dictionary», pp. 1$135,2012$.

[12] A. Tenera, «Contribuição para a melhoria da gestão da incerteza na duração dos projectos através da teoria das restrições», Universidade Nova de Lisboa, 2006.

[13] E. M. Goldratt e J. Cox, The Goal: A Process of Ongoing Improvement, 3rd ed., vol. 3rd Editio. The North River Press Publishing Corporation, 2004.

[14] E. Schragenheim e H. W. Dettmer, Manufacturing at Warp Speed: Optimizing Supply Chain Financial Performance, 1st ed. CRC Press, 2000.

[15] J. Cox III e E. Robinson, «Applying Goldratt's thinking processes to prevent mistakes», Hum. Syst. Manag., vol. 36, n. 4, pp. 315-340, 2017.

[16] H. Lowalekar e R. Ravi, «Revolutionizing blood bank inventory management using the TOC thinking process: An Indian case study», Int. J. Prod. Econ., n. 186, pp. 89-122, 2017.

[17] J. Bauer, A. Vargas, M. Sellitto, M. Souza, e G. Vaccaro, «The thinking process of the theory of constraints applied to public healthcare», Bus. Process Manag. J., 2019.

[18] T. Librelato, D. Lacerda, L. Rodrigues, e D. Veit, «A process improvement approach based on the value stream mapping and the theory of constraints thinking process», Bus. Process Manag. J., vol. 20, n. 6, pp. 922-949, 2014. 
$140 \quad$ M. Gaspar et al.

[19] H. W. Dettmer, «The logical thinking process a systems approach to complex problem solving». 2007.

[20] H. W. Dettmer, Goldratt's Theory of Constraints: A Systems Approach to Continuous Improvement. ASQC Quality Press, 1997.

[21] M. Saunders, P. Lewis, e A. Thornhill, Research methods for business students, 5 th ed. Pearson Education, 2009.

[22] C. Robson, Real World Research: A Resource for Social Scientists and PractitionerResearchers, 2nd ed. Oxford: Blackwell, 2002.

[23] T. Burton-Houle, Field guide to the Theory of Constraints Thinking Process. New Haven, CT: Goldratt Institute, 2000. 